\title{
Factors influencing readability of rapidly presented text segments
}

\author{
THOMAS G. COCKLIN, NICKLAS J. WARD, HSUAN-CHIH CHEN, and JAMES F. JUOLA \\ University of Kansas, Lawrence, Kansas
}

\begin{abstract}
Experiment 1 compared paragraph comprehension for texts shown either as normal pages on a computer terminal screen or as rapid serial visual presentations (RSVPs) of small text segments to a common location. Over several days of practice, reading comprehension was equivalent in the normal presentation mode and the RSVP format. When successive RSVP segments contained some information in common, to mimic the experience of successive parafoveal and foveal views of words in normal reading, comprehension was somewhat worse than when successive segments contained no overlapping information. Experiment 2 used a variety of segment size and segment duration combinations to investigate the optimal means of presenting text in the RSVP format. Across a variety of presentation rates and text difficulties, comprehension was maximal for segments averaging about 12 character spaces in length. In Experiment 3, texts were divided into short idea units or into random segments of equal average length. Comprehension was shown to be greater in the structured condition than in the random condition. An optimal means of presenting text in the RSVP format could be superior to normal presentation methods for reading and other text-processing tasks.
\end{abstract}

There are few methodologies that enable the study of ongoing reading processes without at the same time interfering with normal reading behavior. One such methodology consists of unobtrusively monitoring a reader's eye movement patterns. Eye movement studies have found that as a person reads a text he or she fixates parts of the text for varying amounts of time. The variability of fixation durations could reflect changing processing loads as a reader progresses through a text (Just \& Carpenter, 1980). However, simply monitoring eye movements and fixation durations does not enable experimental control of the parts of text processed by a reader, the sequence of words a reader fixates, or the durations for which words are fixated. Rather, these are regulated by the reader and are, in fact, the variables measured in eye movement studies. Experimental control over what is seen and for

The research reported herein was performed pursuant to Grant G-78-0179 from the National Institute of Education, Department of Education, to the fourth author. However, the opinions expressed herein do not necessarily reflect the position or policy of the National Institute of Education, and no official endorsement by the National Institute of Education should be inferred. This research was also supported by grants from the Biomedical Sciences and General Research Funds of the University of Kansas. Nicklas Ward was supported in part by an NIMH National Research Service Award to the University of Kansa and is now at Johnson Wax, Brantford, Ontario, Canada. Hsuan-Chih Chen is now at the Chinese University of Hong Kong, Shatin, N.T., Hong Kong. We thank Cindy Ikenaga for assistance with Experiment 1 , Wade Welch and Michael Granaas for assistance with Experiment 3 , and Michael Granaas, Tim McKay, Richard Ferraro, Mary C. Potter, and Keith Rayner for comments on an earlier version of this paper. Experiment 3 was based in part on a master's thesis submitted to the University of Kansas by the first author. Requests for reprints should be sent to James F. Juola, Department of Psychology, Fraser Hall, University of Kansas, Lawrence, KS 66045. how long is critical if researchers are to assess how information in text is used in readers' comprehension processes.

One procedure that enables stimulus control for the study of reading is the rapid, serial visual presentation (RSVP) technique (Forster, 1970; Gilbert, 1959). The RSVP procedure consists of briefly presenting successive text segments to a fixed visual location so that the need to move the eyes while reading is eliminated. In previous studies using RSVP techniques, text segments of one or more words have been displayed for about 50 to $600 \mathrm{msec}$ each. Researchers using these procedures have found that the novel RSVP display format does not greatly disrupt reading processes, and that RSVP might encourage more efficient reading for comprehension for some readers in some conditions (Bouma \& de Voogd, 1974; Chen, 1982; Forster, 1970; Juola, Ward, \& McNamara, 1982; Potter, Kroll, \& Harris, 1980; Raygor, 1974; Ward, 1981).

An exception to the general finding of equivalent comprehension in RSVP and normal reading has recently been reported by Masson (1983). He compared reading comprehension in RSVP and normal page formats using short passages from Reader's Digest. In RSVP conditions, the passages were presented one word at a time to a common location on a cathode ray tube (CRT) display with no intervening pauses between successive words or sentences. The successive words were presented briefly $(160 \mathrm{msec}$ or less) to produce RSVP rates of from 375 to 700 words per minute (WPM). In normal reading, or skimming, conditions, the passages were presented as single pages on the display screen and were to be read in amounts of time limited to correspond to the rates used in the RSVP conditions. In three experiments, Masson showed RSVP to 
be inferior to normal reading in his readers' ability to answer detailed or general questions after reading the passages, in their ability to provide summaries covering important ideas, and in their ability to answer questions given prior to presentation of the passages.

Masson's (1983) reported disadvantage for RSVP could be attributed to several differences between perceptual and cognitive aspects of reading in the RSVP and page format that were accentuated by his procedures. In normal reading, each saccadic eye movement carries useful information from one or more words into central vision, where it is processed for an average of $250 \mathrm{msec}-$ the duration of a typical fixation. In Masson's experiments, displays of single words had to be presented at a rate of more than 5 per second in order to be equivalent to a fastnormal reading rate of over $300 \mathrm{WPM}$. Although people can obtain much information from rapid, successive displays, performance inevitably decreases with increasing presentation rate due to the brevity of the displays and subsequent effects of visual masking (e.g., Fischler \& Bloom, 1980; Juola et al., 1982; Lawrence, 1971; Potter, 1983). It is further known that, in normal reading, some time is used at the end of sentences and other linguistically defined constituents for integration processes important for sentence comprehension (e.g., Aaronson \& Scarborough, 1977; Just \& Carpenter, 1980; Mitchell \& Green, 1978). Indeed, Masson demonstrated in two final experiments that RSVP reading could be significantly improved by adding pauses between sentences, and, when this was done, comprehension was no longer significantly worse for RSVP than for text skimmed at the same rate with normal eye movements. In previous studies comparing the readability of texts presented in RSVP and normal page formats, reading comprehension has been shown to be no worse, and sometimes better (see Raygor, 1974), for RSVP displays. In these studies, attempts have been made to improve RSVP reading by displaying more than 1 word at a time so that successive displays could be presented for longer times while a rapid rate of presentation was maintained (e.g., Bouma \& de Voogd, 1974; Gilbert, 1959), by using pauses or other delays at sentence boundaries to facilitate sentence integration processes (e.g., Potter et al., 1980; Ward, 1981), or by doing both (e.g., Chen, 1982; Juola et al., 1982).

Although RSVP might not disrupt readers' comprehension processes, it could encourage reading strategies that differ from those employed when conventionally displayed text is being read. One way to minimize possible differences in readers' strategies is to equate the visual input conditions as much as possible in conventional and RSVP reading. Since readers have considerable experience with extracting information from fixations, using RSVP displays that mimic the duration and useful extent of the visual field during an eye fixation might optimize subjects' performance when they are reading RSVP text. Also, giving readers extensive practice with the novel RSVP procedures and allowing them time for normal sen- tence integration processes might encourage better performance.

Rayner and McConkie (reviewed in Rayner, 1978, 1983) studied the visual span from which useful information is obtainable in single eye fixations. They found that useful vision for reading occurs within a region encompassing about 20 character spaces around the point of fixation. For average adult readers, this field ranges from about 3-4 spaces to the left of the central fixation point to about 15 spaces to the right of fixation. Within these bounds, readers can extract meaning from about the leftmost 10 character spaces surrounding the point of regard. From the rightmost 10 letter positions, readers are able to extract information pertaining only to the size and shape of words that are present. Rayner suggests that readers can obtain meaning only from the word or words centrally fixated, but they might preprocess some graphemic aspects of words immediately adjacent and to the right of the ones fixated. Such preprocessing could facilitate the integration of information across fixations, thus giving rise to the normal experience of continuous reading of text rather than a halting word-by-word process (McClelland \& O'Regan, 1981; Raynor, McConkie, \& Ehrlich, 1978; Rayner, McConkie, \& Zola, 1980). Alternatively, such information might be used mainly to guide successive saccadic movements, and thus be of less importance for RSVP reading.

In the experiments reported here, we compared the effects of RSVP and normal, but time-limited, text presentation formats on readers' comprehension. In Experiment 1, subjects participated in four sessions, each occurring on separate days. In the first session, subjects were given training on the RSVP mode of text display. RSVP conditions were chosen to maximize readability by including one or more words in each successive display and by inserting pauses between sentences. In the second and third sessions, subjects read conventionally displayed texts along with several types of RSVP displays. In the fourth session, some of the RSVP segments included overlaps, such that a particular portion of text occurred two or more times in successive displays. If successive views of a given text segment are useful in normal reading, one might be able to find an overlap condition in which RSVP reading is enhanced due to multiple chances for processing individual words over several simulated eye fixations. On the other hand, if parafoveal and peripheral information available during normal fixations is useful only for guiding subsequent eye movemens, rather than for integration, overlapping information might not be useful for RSVP reading.

In a second experiment, we explored other RSVP variants in a search for optimal ways to present text in this format. The two major parameters of RSVP displays, the size of the text segment shown at any one time and the duration for which it is shown, were traded off against one another. In this way it was possible to present text at a controlled rate and compare comprehension perfor- 
mance across a range of size and duration variables. It is likely that there is an optimal segment size for a given rate of presentation, and it will be important to determine this optimum if the readability of RSVP text is to be assessed adequately.

A third experiment used text segments of an optimal average size as determined by the results of the second study. These segments were constructed either to be as close as possible to the defined optimum, without words' being truncated, or they were constrained to include "idea units," and thus varied greatly in length across individual segments as determined by linguistic structure. In both the fixed-size (random) and idea-unit (structured) conditions, mean segment sizes were the same, and comprehension levels were compared to determine whether regularity in the visual extent of the text segments or the linguistic structure of the segments was more important in influencing readability. All three experiments were designed to develop an optimal means of presenting text in the RSVP format in order to determine whether it might be superior to the page format in reading for comprehension and other text-processing tasks.

\section{EXPERIMENT 1}

\section{Method}

Subjects. Twenty-four University of Kansas undergraduate and graduate students volunteered or received course credit for participation. All were native English speakers and reported normal or corrected-to-normal vision.

Materials. One hundred and twelve paragraphs averaging about 180 words in length were used. Twenty-four paragraphs were selected from the Nelson-Denny (1976) reading inventory, forms A, B, C, and D. The remaining 88 paragaphs were obtained from the $\mathrm{McC}$ Call-Crabbs $(1950,1961)$ test series, books A, B, C, and D. The Nelson-Denny test focuses on evaluating reading performance at the high-school level, whereas the McCall-Crabbs inventory is used to measure comprehension at junior-high-school and upper elementary reading levels. These texts will be referred to as "secondary" and "intermediate" paragraphs, respectively.

Each paragraph was paired with a set of four four-alternative multiple choice questions. In selecting the questions and alternative answers from the test materials, care was taken to ensure that a subject could not readily answer the questions without first reading the paragraph. The paragraphs most similar in length and relative difficulty were assigned for use on Sessions 2, 3, and 4 . The remaining paragraphs (generally the shorter and simpler ones) were assigned to Session 1 . To verify that the questions were acceptable measures of comprehension, a baseline rate of correct responding for each set of paragraphs was obtained from 31 undergraduates who answered the questions without having the benefit of reading the paragraphs. They correctly answered $37 \%$ and $35 \%$ of the questions for intermediate and secondary paragraphs, respectively.

Apparatus. All paragraphs and questions were displayed in normal upper- and lowercase letters on the screen of a Teleray CRT terminal. From the subjects' viewing distance of slightly less than $\mathrm{I} \mathrm{m}$, a sixletter word subtended about $1.5^{\circ}$ of visual angle. The terminal was controlled through a direct interface with a PDP-11/03 minicomputer.

Procedure. Each subject participated in four 1-h sessions on consecutive days. In each session, the subjects were advised of the procedures for that session and were asked to read each paragraph and to answer the subsequent questions to the best of their abilities.

The paragraphs were displayed in two formats. In a page format, paragraphs were displayed in their entireties for fixed time periods and then erased. In the RSVP (window) format, the paragraphs were sectioned into segments one or more words long. These segments were then sequentially displayed one at a time to a common central locus on the CRT screen. In the window condition, sectioning was achieved by using a computer program that scanned the text for a fixed number of characers and then found the nearest space. The text was segmented at that location, and the search for the next segment was begun. In this way, windows of a desired average number of character spaces could be displayed without any words' being truncated. After each sentence, the program inserted a blank window

In the page format, each paragraph was preceded by an instructional cue for $1.5 \mathrm{sec}$ at the upper left corner of the CRT screen, indicating the relative speed at which the subject would have to read (i.e., "Read Normally," "Read Rapidly," or "Read Very Rapidly") in order to complete the text. Following the cue, the CRT screen was filled with overlapped Xs and Os for .5 sec, which were then replaced by the paragraph. Immediately following the paragraph, the mask of Xs and Os was again presented for $.5 \mathrm{sec}$. In the window condition, no instructional cue was given, but each paragraph was preceded and followed for $.5 \mathrm{sec}$ by a mask consisting of a single horizontal line of $31 \mathrm{Xs}$ and Os. The mask occurred in the central region of the CRT screen where the text segments were displayed, and each text segment appeared indented one character space from the leftmost part of the mask. For both page and window conditions, questions pertaining to the paragraph were individually presented after the second mask. The subjects were given unlimited time in which to read and respond to each question. The subjects indicated their response selection by pressing one of the keys labeled $A, B, C$, or D on the terminal keyboard. If on any question the subject's response was incorrect, the word "ERROR" was displayed. If the subject's response was correct, either the next question was displayed or the next trial was begun.

Session 1: Twenty-eight intermediate-level paragraphs were selected for use in Session 1. The paragraphs were assigned randomly to one of two window conditions, in which the paragraphs were sectioned into segments of approximately 5 or 10 characters in length (i.e., on the average, either one or two words per window were displayed in the respective conditions). All segments were displayed for $300 \mathrm{msec}$ each, or slightly longer than the average fixation duration in normal reading.

Session 2: In the second session, each subject read a block of paragraphs in the window format and a block of paragraphs in the page format in a counterbalanced order. In the window block, paragraphs were sectioned into segments averaging 5,10 , or 15 characters in length. These segments were shown for either 200 or $300 \mathrm{msec}$ each to bracket the average fixation duration in normal reading. In the page condition, paragraphs were shown in their entireties for times selected to span the range of viewing times used in the window condition.

Twenty-four new intermediate-level paragraphs were selected for stimuli. Counterbalancing between subjects assured that all paragraphs were shown equally often in page and window conditions. Prior to each block of trials, the subjects were given four paragraphs for practice with the appropriate procedure and modes of text display.

Session 3: The third session replicated the second session with a set of 24 intermediate paragraphs and a set of 24 secondary paragraphs, whereas the subjects read only intermediate paragraphs in Session 2. Thus, the experimental design for the window condition was number of characters displayed $(5,10$, or 15), by display time ( 200 or $300 \mathrm{msec}$ ), by paragraph difficulty (secondary, intermediate). For the page condition, the design was reading time (normal, rapid, or very rapid) by paragaph difficulty. Between subjects, each paragraph occurred equally often in each condition. Prior to each block of trials, the subjects were given a sequence of four paragraphs for practice.

Session 4: In the fourth session, paragraphs were presented with the window display format only. However, unlike in previous sessions, some of the text segments overlapped. That is, the rightmost portion of any window reappeared shifted to the left in subsequent windows. The text segments averaged 10,20 , or 30 characters in length and were displayed for 200 or $300 \mathrm{msec}$ each. No text segments overlapped in the 10 character display conditions. In the 20-character display condition, the segments either did not overlap or overlapped by 10 characters. The 30-character displays had 0-, 10-, or 15-character overlaps. Thus, there were a total of 12 display conditions. As in previous sessions, the sub- 
jects practiced on a series of four paragraphs prior to reading the experimental paragraphs. The same set of 24 intermediate and 24 secondary paragraphs from the third session were used in the fourth session, but no individual subject read the same paragraphs in the third and fourth sessions. Paragraphs were otherwise assigned in a balanced way to all display conditions in Sessions 3 and 4.

\section{Results}

For all subjects, experimental conditions, and paragraphs, the percentages of correctly answered questions were determined. Also, the word-per-minute display rate for each paragraph in each experimental condition was determined by dividing the number of words in the paragraph by its total display time. Session 1 familiarized the subjects with the procedures and equipment and used materials of a lower level than later sessions. Therefore, the data from Session 1 cannot be compared with those from the other sessions, and will not be discussed.

Figure 1 summarizes the data from Sessions 2 and 3. In each of the RSVP and page conditions, the subjects' abilities to answer comprehension questions declined as experimentally imposed reading rates increased. In Session 2, the subjects' comprehension scores declined from about $80 \%$ correct at $215 \mathrm{WPM}$ to $51 \%$ at $730 \mathrm{WPM}$. These results were replicated in Session 3, in which performance ranged from $85 \%$ to $45 \%$ correct. However, overall performance was significantly greater for intermediate paragraphs in Session 3 than in Session 2 [t(23) $=2.54, \mathrm{p}<.05$ ]. In Session 3, performance was better for intermediate than for secondary paragraphs $[\mathrm{t}(23)$ $=7.5, \mathrm{p}<.01]$. No other results, including all comparisons between page and RSVP conditions, were significant.

Figure 2 outlines the data from Session 4. As in prior sessions, performance declined as word-per-minute rate

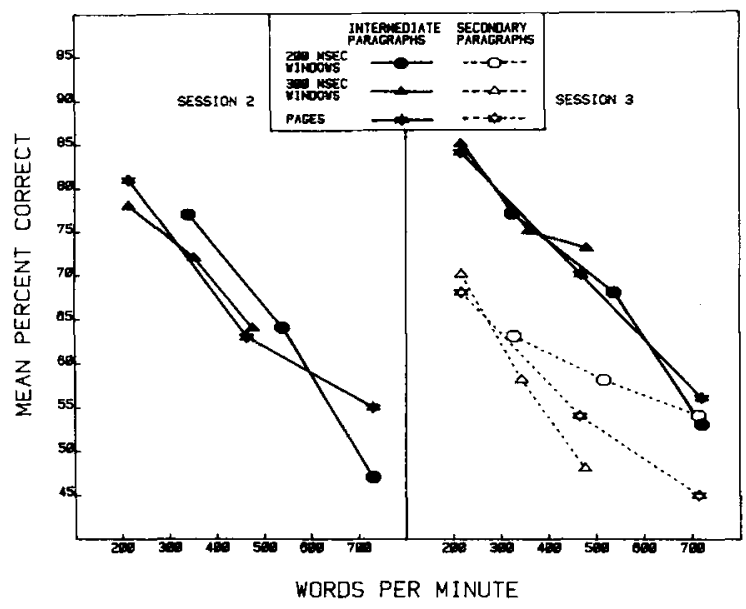

Figure 1. Mean percent correct comprehension score for intermediate and secondary paragraphs for 200- and 300-msec window conditions and page conditions plotted against words per minute. The connected points represent (from left to right) window sizes of 5,10 , or 15 character spaces in RSVP conditions and normal, rapid, or very rapid reading instructions in page conditions (Experiment 1, Sessions 2 and 3).

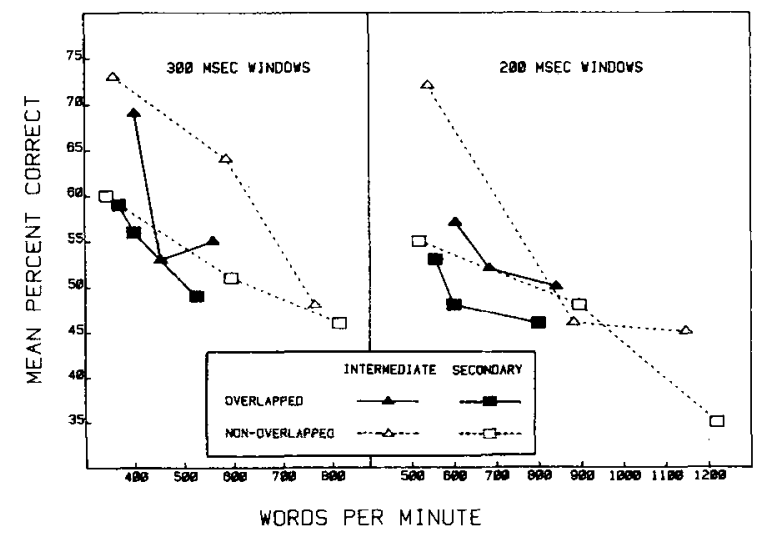

Figure 2. Mean percent correct comprehension score for intermediate and secondary paragraphs for overlapped and nonoverlapped, 200- and 300-msec window conditions plotted against words per minute. The connected points represent (from left to right) window sizes of 10, 20, or 30 character spaces in the nonoverlapped RSVP conditions and window sizes of 20 characters with 10-character overlaps, 30 characters with 10-character overlaps, and 30 characters with 15-character overlaps in the overlapped RSVP conditions (Experiment 1, Session 4).

increased. In the nonoverlapping conditions, mean percent correct responses declined from about $73 \%$ at $325 \mathrm{WPM}$ to $35 \%$ at 1,200 WPM. In overlap conditions, comprehension performance declined similarly over a smaller range of presentation rates. Comprehension performance was $18 \%$ better for intermediate paragraphs than for secondary paragraphs $[\mathrm{t}(23)=3.28, \mathrm{p}<.01]$.

In order to assess the effects of overlapped text, the comprehension scores were collapsed for the three overlap conditions in each window duration $\times$ text difficulty combination. These four means were then compared with the means for the nonoverlapping window size 10 and 20 conditions for the same four cells. A 2 (duration) $\times 2$ (difficulty) $\times 2$ (overlap vs. nonoverlap) ANOVA revealed significant effects of window duration $[F(1,23)=$ $6.96, \mathrm{p}<.01]$ and difficulty $[\mathrm{F}(1,23)=9.63, \mathrm{p}<.01]$. The advantage for nonoverlapped RSVP displays was marginally significant $[F(1,23)=3.04, p<.09]$. The latter is more impressive when it is noted that the mean word-per-minute rate was higher in the nonoverlapped conditions used in the comparison (471 and 710 WPM for the 300- and 200-msec-duration conditions, respectively) than in the overlapped conditions (451 and 678 WPM, respectively). When each subject's mean percent correct score was found for the comparable overlapped and nonoverlapped conditions, the 7\% disadvantage in comprehension for the overlapped conditions was significant $[\mathrm{t}(23)=2.54, \mathrm{p}<.05]$. We conclude that comprehension performance is reliably worse in the overlap conditions used than in RSVP conditions without overlaps if overall presentation rate is equivalent.

\section{Discussion}

Although significant practice effects were observed between Sessions 2 and 3, they applied equally to both RSVP 
and conventional reading situations. The absence of any differences in Sessions 2 and 3 between window and page conditions suggests that a few hours' practice in reading the novel RSVP displays is not sufficient to demonstrate advantages for RSVP over normal reading, if indeed such advantages can be demonstrated at all. Comprehension performance was shown to be highly sensitive to rate of presentation and text difficulty in both conditions. These results indicate that (1) the measures of comprehension used have some validity and are sensitive enough to pick up real differences in comprehension when such differences exist, and (2) it is likely that comprehension processes in normal and RSVP reading are very similar. The absence of differences in the window-page comparison for intermediate and secondary paragraphs indicates that people find RSVP text no more difficult to comprehend than they do conventionally displayed texts. Thus, in general, eye movements are not required for people to read with normal levels of comprehension. In fact, removing the need for eye movements might lead to superior performance in some cases (Bouma \& de Voogd, 1974; Chen, 1982; Gilbert, 1959; Juola et al., 1982; Potter et al., 1980; Raygor, 1974).

At first it might appear to be counterintuitive that the elimination of normal eye movement patterns and variations in fixation durations could in any way be conducive to reading efficiency. Normal deviations from an invariant scanning pattern have been argued to reflect changes in processing load or decisions about how to sample information from text (e.g., Just \& Carpenter, 1980; LevySchoen \& O'Regan, 1979; McConkie, 1979). Thus, such variations in saccadic extent and fixation duration are thought to be necessary concomitants of normal reading processes, much as are regressive eye movements and pauses, none of which are captured in the methodical RSVP format. Because the present results and most of the previous research are consistent in showing that reading comprehension is no worse for RSVP displays than for normal pages of text, any cost due to the invariance of display parameters in RSVP must somehow be compensated by benefits due to the elimination of normal eye movement patterns.

It is possible that typical eye movements are inefficient because normal readers tend to be conservative, moving their eyes in smaller forward saccades and in more frequent backward or regressive movements than are necessary for clear resolution of the text (Crowder, 1982; Hochberg, 1976). Eye movements themselves sometimes result in inaccurate fixation locations, particularly in return sweeps to new lines of text, necessitating corrective eye movements. Furthermore, vision during saccadic eye movements is suppressed (see Volkmann, 1976), beginning about 50 msec before the onset of each saccadic movement and continuing for about $100 \mathrm{msec}$ after the eyes are again at rest. Thus, visual information input might be significantly reduced in normal reading relative to RSVP reading due to saccadic suppression. Rayner, Inhoff, Morrison, Slowiaczek, and Bertera (1981) showed that information obtained in the first $50 \mathrm{msec}$ of a fixation is sufficient to promote normal reading, prompting O'Regan (1983) and Wolverton and Zola (1983) to suggest that saccadic suppression is not an important factor in normal reading. Yet Breitmeyer (1983) and Kolers (1983) argued for its existence in above-threshold contrast situations, although saccadic suppression might indeed be weaker in normal reading than in the threshold detection studies in which it was first investigated.

A final inefficiency and potential problem with normal reading lies in the fact that although the eye guidance system subjectively appears to be automatic, some cognitive processing capacity must unavoidably be devoted to the control of eye movements and to the determination of fixation duration. This demand for cognitive resources could diminish the capacity available for other processes such as the main task of reading for comprehension. Thus, there is at least potential for discovering new methods of presenting text such that the need for eye movements is eliminated and at the same time reading efficiency is improved.

In normal reading, the span of useful vision generally exceeds the length of the sacccade, so that words are visible to some extent in several successive fixations. Information obtained in the parafovea could be useful for at least two reasons: (1) for preliminary analysis and subsequent integration with information obtained in foveal vision, and (2) for planning or directing subsequent eye movements (Rayner, 1978). The results from Session 4 demonstrated that overlaps in RSVP conditions were not helpful and, in fact, generally disrupted reading comprehension relative to RSVP conditions with equivalent wordper-mintue rates but without overlapping information across successive windows. This result provides no support for the idea that information present in parafoveal vision is used for preprocessing text in a way that aids subsequent foveal analysis. This conclusion must be qualified by the differences that exist between overlapping information in the RSVP task and successive parafoveal and foveal views of text in normal reading. The RSVP overlaps only approximate the way that normal saccadic movements provide multiple glimpses of the same sections of text. In normal reading, saccadic movements presumably follow the flow of attention to the point in the text from which additional information is needed (e.g., McConkie, 1979). Furthermore, the lack of subjective control and subsequent expectations of how the text should appear after each new window in RSVP might have led to confusions and greater difficulty in reading the text. In studies in which subjects have had good expectations about where successive stimuli will occur, prior to parafoveal information has been shown to facilitate subsequent naming times for foveally presented words (McClelland \& O'Regan, 1981; Rayner et al., 1978). Rayner, Well, Pollatsek, and Bertera (1982) have also strongly argued that, in normal reading, parafoveal information is used to facilitate processing of foveally fixated words on subsequent fixations. It is clear from the present data, 
however, that the attempt to provide peripheral and parafoveal information successively in simulated eye fixations was not successful in improving RSVP reading.

In summary, the results of Experiment 1 demonstrated that RSVP reading and normal reading can result in similar levels of comprehension and that comprehension processes themselves might be largely invariant over a wide range of textual input formats. The attempt to improve RSVP readability by simulating one aspect of normal eye fixation data-the overlap of useful vision across successive views of the text-was not successful. It could well be the case that our search for optimal RSVP conditions will lead us away from mimicking normal eye movement behavior, and, if so, scanning a text with typical eye movements might not be the best way to read for comprehension (Ward, 1981; Ward \& Juola, 1982).

\section{EXPERIMENT 2}

In Experiment 1, window size and window duration were crossed to produce a variety of presentation rates in RSVP conditions. Both parameters were confounded with presentation rate, because rate increased directly with window size and inversely with window duration. Such tradeoffs prevent independent analyses of the effects of window size and duration on the readability of RSVP text. In Experiment 2, the word-per-minute rate of presentation was fixed at four different levels, and window size was traded off against window duration within each level. The aim was to determine whether there is an optimal window size for maximizing the readability of RSVP displays and whether this optimum is the same across a range of presentation rates and text difficulties. Since most previous RSVP research has used single-word displays or allowed window size and presentation rate to be correlated, the second study is a necessary step in determining optimum parameter values for increasing the readability of RSVP displays.

\section{Method}

Subjects. A total of 72 subjects participated in single 80 -min sessions. Forty-four were students from an introductory psychology class who participated for class credit, and 28 were volunteers who were paid $\$ 3.00$ for their participation. All subjects were native English-speaking students at the University of Kansas who reported normal or corrected-tonormal vision. None of them had participated in Experiment 1.

Materials and Apparatus. Twenty-four of the intermediate-level paragraphs and the 24 secondary-level paragraphs used in Experiment 1 were used in the present study. All were accompanied by the same comprehension questions as in Experiment 1. The apparatus and viewing conditions were the same as those used in Experiment 1.

Procedure. It was decided to vary window size from a minimum of exactly one word per window to a maximum average of 20 characters per window, or about three to four words. This range should span the limits of useful vision during a single fixation in normal reading (e.g., Rayner, 1978, 1983). A trend analysis of the relation between comprehension and window size would then be of interest, with a significant linear trend indicating that either small or large windows are optimal, whereas a significant quadratic trend would indicate that an intermediate window size is optimal.

The text-sectioning program from Experiment 1 was used to break the 48 paragraphs into units varying from a minimum of one word per window to a maximum of about 20 character spaces per window. Each
Table1

Mean Window Durations (in Milliseconds) for Each of the Six Window Size Conditions Used to Produce Reading Rates of 200, 400, 600, or 800 Words per Minute (WPM) (Experiment 2)

\begin{tabular}{lrrrrrr}
\hline & \multicolumn{6}{c}{ Mean Number of Character Spaces per Window } \\
\cline { 2 - 7 } WPM & 5.9 & 6.7 & 8.2 & 9.9 & 12.6 & 19.6 \\
\hline 200 & 303 & 352 & 420 & 519 & 682 & 992 \\
400 & 152 & 176 & 210 & 260 & 341 & 496 \\
600 & 101 & 117 & 140 & 173 & 236 & 331 \\
800 & 76 & 88 & 105 & 130 & 170 & 248 \\
\hline
\end{tabular}

paragraph was divided into about six evenly spaced numbers of windows ranging from about 180 (the mean number of words per paragraph) to about 55 windows per paragraph. Within these limits, the actual number of character spaces varied from window to window, since the program was written to select windows as close as possible to a desired length without truncating any words. The mean numbers of characters obtained per window in the six size conditions are shown in Table 1. Also in Table 1 are the mean window durations used in each size condition to produce the four chosen rates of presentation. These durations were individually determined for each paragraph so that rates as measured in words per minute were constant for each paragraph within any cell of the design. The rates used were chosen to span the range from slower than normal to more than twice the normal reading rate.

The combination of six window sizes $x$ four window durations yielded 24 different conditions. Each subject read one intermediate-level paragraph and one secondary-level paragraph in each condition. The assignment of paragraphs to conditions was counterbalanced randomly such that, in the experiment as a whole, each paragraph was used exactly three times in each condition. Twenty-four different randomly ordered paragraph sequences were used for three subjects each.

The experiment began with instructions and three practice paragraphs. Paragraphs were presented one or more words at a time, according to the window size parameter value for that trial. Each trial began with a 500 -msec presentation of a masking field in the center of the CRT screen. The mask consisted of a row of 31 overlapped uppercase Xs and $O s$ and was followed immediately by the first window. Each window was left-justified and indented one space from the edge of the mask. Successive windows followed each other with no interstimulus inter$\mathrm{val}$, and the last window was followed immediately by the masking field.

Each paragraph was followed by four four-alternative forced-choice comprehension questions that also had been used in Experiment 1. They were presented one at a time on the CRT screen, and the subjects responded by pressing the keys A, B, C, or D on the keyboard. Incorrect responses were followed by the word "ERROR" centered on the screen. Correct responses were followed by the next question or.the onset of the masking field before the next paragraph. The subjects were allowed one 10 -min rest period approximately halfway through the experimental session.

\section{Results}

The mean correct comprehension scores for each subject in all cells of the design were determined and analyzed using a three-factor ANOVA. The factors were paragraph difficulty (intermediate vs. secondary), presentation rate $(200,400,600$, or $800 \mathrm{WPM})$, and mean window size $(5.9,6.7,8.2,9.9,12.6$, or 19.6 characters per window). The analysis found significant main effects for paragraph difficulty $[\mathrm{F}(1,71)=214.2, \mathrm{p}<.001]$ and presentation rate $[\mathrm{F}(3,213)=146.4, \mathrm{p}<.001]$. The window size factor was only marginally significant $[F(5,355)=1.86, p<.10]$. The only significant interaction involved paragraph difficulty and presentation rate $[F(3,213)=4.34, p<.01]$. As can be seen in Figure 3, 
comprehension decreased with presentation rate and paragraph difficulty, and the interaction probably is due to a slight floor effect for the highest presentation rates.

Although the window size effect was only marginally significant, the planned trend analysis was carried out. This analysis was justified not only on the basis of the a priori expectation that windows larger than one word but limited by the perceptual span would be optimal for RSVP reading, but also on the basis of the observation that seven of the eight plots in Figure 3 show a peak between the two extreme window sizes. The eighth, that for the least difficult condition, might be artificially flattened due to ceiling effects. Therefore, the first and second polynomial trend components relating percent correct to mean window size were tested with the main goal of determining if any optimal window size could be found and whether this optimum varied with text difficulty or presentation rate. Only the quadratic trend was significant $[F(1,71)=5.77, p<.05]$, and there were no significant interactions among the window size trends and the factors of text difficulty and presentation rate. Since the quadratic trend was statistically uniform across all conditions, the data were collapsed across difficulty and rate, as shown in Figure 4 . The best-fitting quadratic function plotted in Figure 4 accounts for more than $75 \%$ of the variance in the comprehension scores and has the equation $\mathrm{Y}=54.10+1.42 \mathrm{X}-0.06 \mathrm{X}^{2}$. It has a maximum at a window size of 12.1 characters.

\section{Discussion}

The results demonstrated that not all window size conditions are equivalent for text readability in the RSVP format. Over the range of text difficulties and presentation rates investigated here, we found that windows averaging about 12 character spaces in length were optimal for reading comprehension. This result is interesting in that most previous studies of RSVP reading have presented texts one word at a time (e.g., Masson, 1983; Potter et al., 1980; Raygor, 1974) or have allowed window size and reading rate to be confounded (e.g., Bouma \& de Voogd,

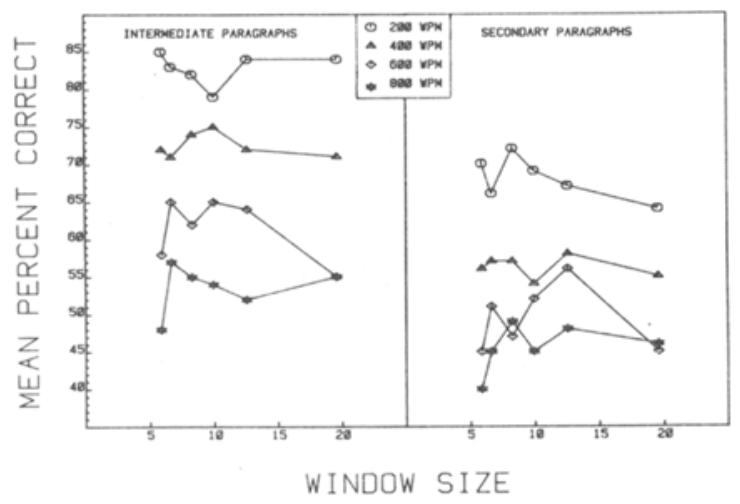

Figure 3. Mean percent correct comprehension scores for intermediate-level paragraphs (left panel) and secondary-level paragraphs (right panel) plotted against mean window size for the 4WPM presentation rate conditions (Experiment 2).

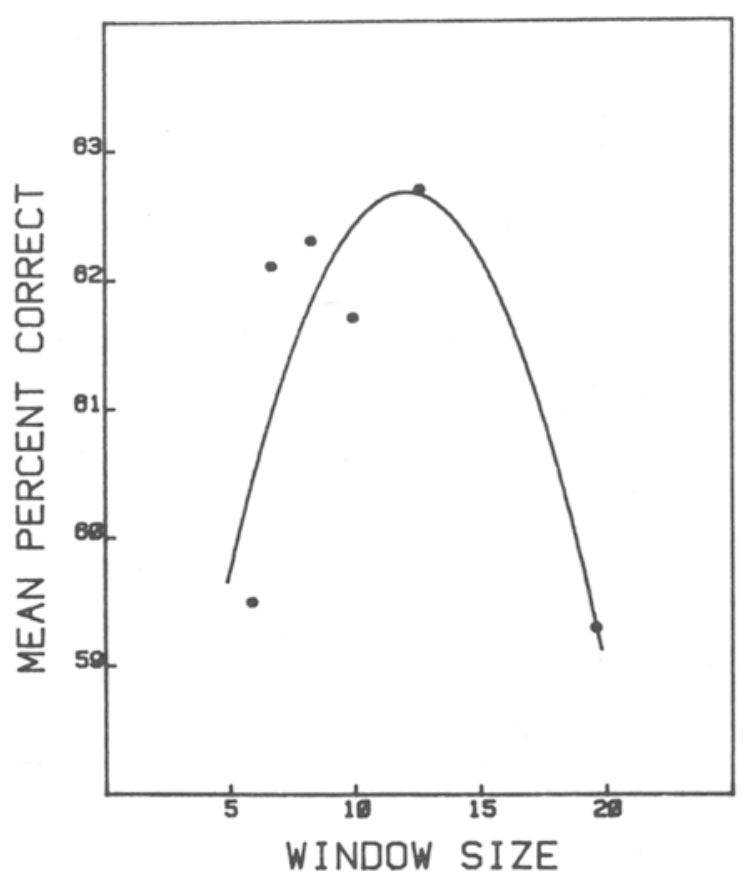

Figure 4. Mean percent correct comprehension scores for all paragraphs and rate conditions combined plotted against mean window size. The curve is the best-fitting quadratic function (Experiment 2).

1974; Juola et al., 1982). Only when these two display parameters are unconfounded can the present results be demonstrated.

One interpretation for the quadratic trend relating comprehension to window size is that there is a tradeoff between two factors: one that positively influences reading comprehension as window size increases and one that negatively influences comprehension with increasing size. Reading is poorly characterized as a word-by-word process, and the preservation of some contextual components of text is a necessary precondition for correct interpretation of a word's meaning. By this argument, increasing the amount of text visible around the point of fixation should continuously improve reading comprehension to some limit, as Rayner and Bertera (1979) showed. In the RSVP conditions used in Experiment 2, however, the subjects generally had time for only a single fixation on each window, and the visual span for obtaining information in reading is increasingly limited in its usefulness to a maximum of about 15 characters to the right of a fixation (e.g., McConkie \& Rayner, 1975). Thus, the peak at about 12 characters in the present study probably results from a combination of the need (or use) of context in reading and the limits of visual acuity or perceptual capacity.

\section{EXPERIMENT 3}

Experiment 2 demonstrated that there is some optimal window size for maximizing the readability of text presented in the RSVP format. Although the trend analysis indicated that about 12 characters (or two or three 
words) are optimal, a more interesting question is why windows of intermediate size should be best, rather than exactly what the optimal size might be. Our interpretation is that both perceptual and higher order cognitive and linguistic factors are operating to favor windows of intermediate size. Experiment 3 was designed to test the relative importance of these two factors in determining the readability of text parsed into multiword windows in the RSVP format.

One hypothesis for the 12-character maximum is that visual acuity and/or a short-term visual buffer is limited in capacity to about 12 character spaces. Letters and words within this range are clearly perceived and held briefly in memory during lexical access and semantic interpretation. If successive windows are larger than the buffer capacity, some information is necessarily lost. On the other hand, if successive windows are smaller than the buffer capacity, the result could be an inefficient underutilization of resources. Thus, the optimum at about 12 characters could be due to matching the information input load to the processing capacity of the visual system (see Bouma \& de Voogd, 1974, McConkie, 1979, Potter, 1982, 1983, and Potter et al., 1980, for similar ideas).

An alternative to this perceptual hypothesis is the idea that words are chunked into small groups at an early processing stage in reading, such that the context in which a word appears is used to facilitate lexical access and interpretation of its meaning. The presentation of two- to three-word windows facilitates the reading process by preserving some useful context that is used in the immediate process of interpretation. Obviously, if this is the case, interpretation of words and comprehension of text could be improved in RSVP reading if successive windows were chosen specifically to contain useful contextual entities such as "idea units" based on phrase structure or other linguistic analyses. The presentation of text in idea-unit chunks rather than as isolated words or as normal continuous text has been shown to be a comprehension aid in a number of studies (e.g., Cromer, 1970; Martinez, Ghatala, \& Bell, 1980; O'Shea \& Sindelar, 1983). Although skilled adults are usually indifferent to such manipulations, less skilled readers and children generally benefit from parsing text into phrases or idea units. The RSVP situation is different from normal reading, however, in that, rather than the reader's selecting, with his or her own eye movement patterns, which segments of text to process, the text is always parsed into successive chunks for the reader. This selectivity is evident from eye fixation studies such as those of Just and Carpenter (1980; Carpenter \& Just, 1983), who have reported that readers of scientific passages fixated about $83 \%$ of content words and only $38 \%$ of function words. Furthermore, Ehrlich and Rayner (1981) demonstrated that high semantic constaints can diminish the probability that a content word is fixated in normal reading. Thus, dividing a text into idea-unit chunks that generally begin with function words or modifiers and end with major noun or verb content words could improve RSVP reading for several rea- sons. The processing resources devoted to parsing a text into idea units could be freed for other processes related to comprehension, and the maintenance of information from successive RSVP displays could be facilitated by making them meaningful units rather than splitting them across linguistically defined clause and phrase boundaries. This latter hypothesis would be especially meaningful if buffer or recycling models of reading are relevant for processes of comprehension (e.g., Bouma \& de Voogd, 1974; Kintsch \& van Dijk, 1978; Mitchell \& Green, 1978; Potter, 1983).

Experiment 3 was designed to discover whether perceptual or linguistic factors dominate in determining the readability of text presented as sequences of short units. This hypothesis was tested by comparing reading comprehension for text that had been sectioned into either unstructured units of relatively constant size versus idea units of more variable size. Besides deciding between the two alternative explanations for the results of Experiment 2, the results should clearly indicate how the RSVP method of text presentation can be optimized to promote rapid reading with high levels of comprehension.

\section{Method}

Subjects. Forty University of Kansas undergraduate and graduate students volunteered or were given credit for participation. All were native English speakers and reported normal or corrected-to-normal vision. None of them had participated in Experiments 1 or 2 .

Materials and Apparatus. The 24 intermediate- and 24 secondarylevel paragraphs and corresponding questions used in Experiment 2 were used in the present study. Each paragraph was divided into an equal number of segments by two different procedures: a structured parsing scheme and a random sectioning scheme. In the structured case, four independent judges were instructed to segment each paragraph into short "idea units." Each judge was instructed to divide the text into segments averaging about 12 characters (two or three words) with the constraint that the words included within each segment should represent a single component of the sentence and convey a simple idea. These idea units were not all linguistically defined components of the sentence in that the parsing of the text did not follow an absolute linguistic rule of clause and phrase boundaries. For the intermediate paragraphs, the judges reached $88 \%$ agreement on idea units that averaged 13.00 character spaces in length. For the secondary paragraphs, the judges reached $92 \%$ agreement on idea units that averaged 13.65 character spaces in length.

For the second, or random, condition, a computer program sectioned the text into segments of approximately 13 characters, rounding the number of characters displayed in each window to the nearest word. For the intermediate paragraphs, the average number of character spaces displayed was 13.08 , and for the secondary paragraphs, the average length was 13.52 spaces (see Table 2). Across display formats there was a 16\% agreement among text segments generated for the structured and the random conditions for the intermediate-level paragraphs and $17 \%$ agreement between conditions for the secondary-level paragraphs. Thus, although the two procedures yielded windows that were very simi-

Table 2

Mean, Standard Deviation, and Range for the Number of Characters per Window (Experiment 3) Paragraph Difficulty

\begin{tabular}{llllllll} 
& \multicolumn{4}{c}{ Paragraph Difficulty } \\
\cline { 2 - 4 } \cline { 5 - 7 } Display Format & Mean & SD & Range & & Mean & SD & Range \\
\hline Structured & 13.0 & 4.66 & $3-28$ & & 13.65 & 4.90 & $2-30$ \\
Random & 13.08 & 2.0 & $4-20$ & & 13.52 & 2.24 & $7-21$ \\
\hline
\end{tabular}


lar in average size, they differed significantly enough in length variabiity and structure to allow a test of the perceptual versus linguistic hypotheses.

The apparatus and viewing conditions were the same as in the first two studies. It was decided to approximate a normal reading rate of about 300 WPM, which was accomplished for each paragraph by displaying each successive window for an average duration of about 520 msec. Although a window of average length can be read in its entirety in a single fixation, the 520-msec duration allows time for a second fixation, which might be necessary for longer windows approaching 20 character spaces in length.

Procedure. Each individual participated in a single session that lasted approximately $90 \mathrm{~min}$ and was blocked into two parts. First, the subject was briefed about the difference between the random and structured display formats and then given detailed instructions and four practice paragraphs for either the random or structured condition. Then, a set of 20 paragraphs was presented in a main, uninterrupted trial block that was consistent with the instructions and the practice set. At the end of the first trial block, the subject was given a 10 -min rest and then was given the instructions and four practice paragraphs for the remaining format. After practice, the subject read the second uninterrupted block of 20 paragraphs in the second format condition.

Twenty subjects read the random paragraphs first and then the structured paragraphs, and 20 read the structured paragraphs first. Paragraph difficulty was counterbalanced randomly with the between-subjects factor of display format order. As in the previous two studies, all subjects answered four four-alternative multiple-choice questions about the content of the paragraph immediately after having read each passage.

\section{Results}

The mean percent correct comprehension scores for each subject in all cells of the design were determined and subjected to a mixed factorial ANOVA. The betweensubjects factor was display format order (randomstructured vs. structured-random), and the within-subject factors were paragraph difficulty (intermediate or secondary) and display format (structured or random). Since the format order condition had no significant effects on the results, the data were collapsed across this factor for summarization in Figure 5.

There were main effects of paragraph difficulty $[\mathrm{F}(1,38)$ $=79.12, \mathrm{p}<.001]$ and display format $[\mathrm{F}(1,38)=5.13$, $\mathrm{p}<.05]$. Comprehension was lower for the more difficult paragraphs, and when the windows conformed to linguistic structure, the texts were better comprehended than when they were broken into windows that retained a more consistent length but had no consistent linguistic structure. There were no significant interactions in the analysis. Linguistically parsed texts were comprehended more accurately than randomly parsed texts for both Nelson-Denny (secondary) and the McCall-Crabbs (intermediate) materials.

\section{Discussion}

The results showed that although there might be a perceptual advantage in RSVP reading for windows averaging about 12 character spaces in length, an even greater advantage in comprehension level can be gained if successive windows are chosen to reflect phrase structure variables. Even though the variance of the number of characters per window was over four times as great in the structured condition as in the random condition, ideaunit windows were read more efficiently than unstructured

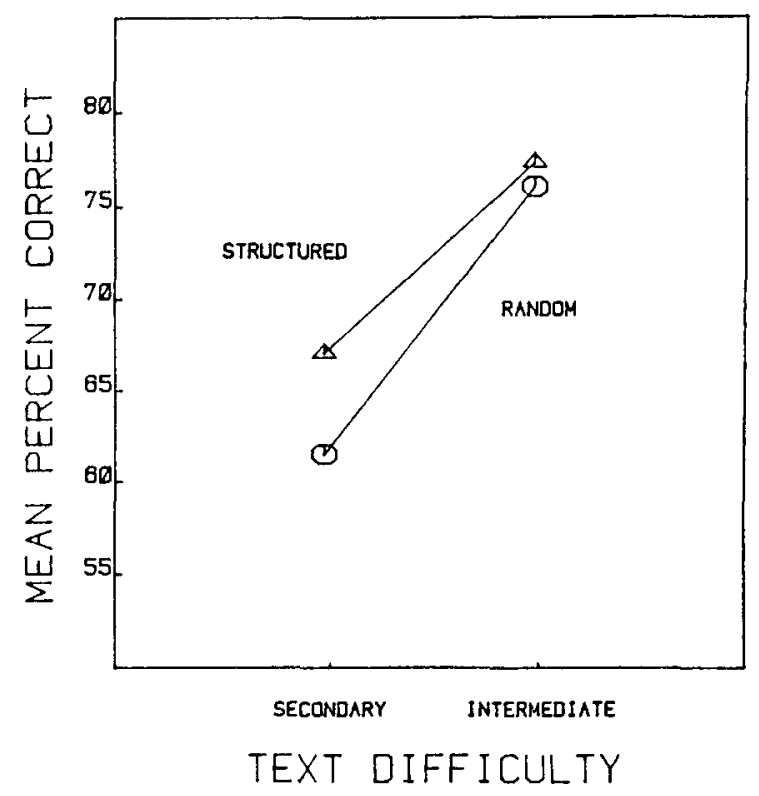

Figure 5. Mean percent correct comprehension scores plotted against paragraph difficulty for the structured and random window conditions (Experiment 3).

windows. This result implies that sentence structures could be used at an early processing level in reading to facilitate perceptual processes such as word identification and lexical access (Aaronson \& Scarborough, 1977; Juola et al., 1982). The beneficial effects of a structured context during text presentation could then enable the reallocation of processing capacity from data-driven word-recognition processes to more conceptually driven processes important for comprehension to proceed efficiently (Rumelhart, 1977). Structural windows could also benefit performance by allowing more efficient encoding and maintenance of idea units or propositions in a conceptual buffer that works to integrate information from successive text segments into a coherent macrostructure (e.g., Kintsch \& van Dijk, 1978). It is conjectural whether the present results would generalize to higher rates of RSVP. At the 300-WPM rate, windows are presented long enough to allow two eye fixations, if necessary, which could assist in reading the longer windows that are more prevalent in the structured condition. At higher rates that would allow only one fixation per window, it is possible that the more consistent size of the windows in the random condition could lead to an advantage over structured windows.

\section{GENERAL DISCUSSION}

The present studies investigated several factors thought to influence the readability of text presented as a sequence of individual words or small groups of words to a single location on a visual display. The goal of this continuing research project is to optimize the readability of text presented in the RSVP mode and to determine whether 
or not the method has any advantages over normal reading.

Experiment 1 replicated earlier results (e.g, Bouma \& de Voogd, 1974; Chen, 1982; Juola et al., 1982; Potter et al., 1980; Raygor, 1974; Ward, 1981) indicating that RSVP reading results in levels of comprehension that are generally no worse than those obtained when text is read normally, with eye movements, at equivalent rates. This was true over several days of practice in which reading comprehension improved in the RSVP case but, somewhat paradoxically, improved equivalently in the normal case. One attempt to make RSVP reading more like normal reading in Experiment 1 was to have information repeated in successive windows. In this way, we tried to simulate the experience of normal reading in which words are first previewed in peripheral and parafoveal vision before they are centrally fixated. This attempt to simulate normal reading with partial overlaps in successive windows was unsuccessful; comprehension was generally worse in the overlap conditions than in nonoverlapping RSVP conditions in which the text was presented at equivalent rates.

Although eye fixation patterns in normal reading typically result in several parafoveal and foveal views of most text segments, this aspect of reading is probably poorly simulated in RSVP. The RSVP overlap conditions result in successive views of text that are not under control of the reader, and thus result in unexpected sequences of visual inputs as well as foveal views of text that might not have been selected had the fixation placement been under the reader's control. In normal reading, parafoveal and peripheral information is used to guide eye movements, and, presumably, readers generate some expectations about how the text should appear in the fixation following a guided movement. For these reasons, it is somewhat surprising that McConkie, Zola, and Wolverton (1980) and O'Regan (1981) found that small shifts of the text to the right or the left during saccades go unnoticed. The shifts of 10 to 15 character spaces in the overlap conditions used in Experiment 1 produced much greater amounts of uncertainty, however, about the relative positions of given text segments in successive, simulated fixations.

A related use of parafoveal information in normal reading is to use some physical, structural, or syntactic markers not only to guide eye movements, but to select a preferred viewing location (e.g., Rayner, 1979, 1983). For single words that are fixated, this tends to be somewhat left of center, whereas highly constrained words (Ehrlich \& Rayner, 1981) and function words (Just \& Carpenter, 1980) tend to be skipped over entirely and fixated less often than other words. In the overlap conditions in RSVP reading, the lack of control over successive glimpses of the text undoubtedly resulted in a second viewing of words that would have been fixated differently or skipped with normal eye movements. Thus, the redundancy of the overlap conditions in RSVP would be less optimal than that resulting from successive views of texts accomplished by eye movement control systems. Under RSVP conditions without overlaps, subjects can maintain a single fixation point that should be close to the preferred viewing location for the first word in each window, and any other words can be processed foveally or parafoveally, depending on their length and the size of the window. Whether it is better to present text one word at a time or in slightly larger windows was investigated in Experiment 2.

In the second study, we controlled the rate of text presentation and investigated the effects of text difficulty and mean window size on RSVP reading. By varying mean window size between about 5 and 20 characters, comprehension was found to peak at window sizes of about 12 character spaces across a range of presentation rates and text difficulties. Rayner (1983) and others have argued that this might be the extreme range over which all letters are reliably identifiable during a single fixation in normal reading. Forcing subjects to read larger windows with a single fixation in the RSVP case would have produced an increase in word encoding and lexical access errors. Similarly, using smaller windows could have limited performance due to an underutilization of perceptual resources available for each new input, or due to the fact that small windows must be presented in a relatively more rapid sequence to achieve a comparable reading rate (Masson, 1983). Rapid sequences of small displays might not be as perceptible as larger displays presented at slower rates, and yet reading rates measured in words per minute would be comparable.

In either case, we know that the perceptual span in normal reading is unlikely to be a constant, but is influenced by momentary changes in perceptual and linguistic factors associated with the region of text being processed (McConkie, 1983). O'Regan (1983, p. 121) argued that the "zone of perceptibility" can be enlarged by the combination of visual and linguistic information such that several words can be processed during a single fixation (see also Hogaboam, 1983, and Rayner, 1983). For these reasons, we thought that reading RSVP text could be improved if windows of an optimal size from perceptual considerations were chosen to be consistent with linguistic structure as well.

This further refinement of the RSVP procedure was achieved in Experiment 3, in which it was shown that if windows included structurally related words rather than random segments from a text, reading comprehension can be improved. As the optimal perceptual parameters of windows are modified to include phrase and clause structures, comprehension improves despite the increased perceptual variance (i.e., number of characters per window). This result is important, since it indicates that most previous studies of text readability using the RSVP format were not using optimal display parameters (e.g., Masson, 1983). It is possible that chunking text into successive idea units for RSVP presentation aids reading comprehension for several reasons. First, as previously argued, the perceptibility of individual displays could be enhanced due 
to the ability of linguistic information to enhance visual information and increase the "zone of perceptibility" (O'Regan, 1983). Second, it is possible that dividing a text into meaningful structural units is a resourcedemanding process that readers might more readily execute in normal reading than in the RSVP case. With the text divided into idea units in RSVP, not only might syntactic analysis be made easier, but the chunking and retention of information in a working-memory buffer might be enhanced. Thus, windows that are constrained to be consistent with text structural variables could enhance perceptual processes, syntactic analysis, and buffering of information in working memory while comprehension and integration are taking place.

From the results of the present studies, we conclude that optimal RSVP conditions should include successive, nonoverlapping text segments of about two or three words in length. When possible, segments of this average size should be chosen to represent integrated ideas or be short phrases from the text. Additional insight into which linguistic elements are useful for optimizing RSVP will lead us to $\mathrm{a}$ better understanding of reading comprehension and integration processes that occur both in reading RSVP displays and in normal reading with eye movements. Since most previous research using relatively unsophisticated RSVP formats has shown little or no difference in comprehension between normal and RSVP modes, it is likely that an improved RSVP format will result in comprehension superior to that obtained from normal reading at equivalent rates. It is certain that, in the future, an increasing percentage of all of what we read will be presented on computer displays. Even a small advantage for the RSVP method of text presentation could have significant consequences for improving reading efficiency and performance in other text-based tasks such as search and retrieval of information from data banks. The method could also have important consequences for computerbased educational programs in beginning and remedial reading instruction.

\section{REFERENCES}

aAronson, D., \& SCarborough, H. S. (1977). Performance theories for sentence coding: Some quantitative models. Joumal of Verbal Learning and Verbal Behavior, 16, 277-303.

BoumA, H., \& DE Voogd, A. H. (1974). On the control of eye saccades in reading. Vision Research, 14, 273-284.

BREITMEYER, B. G. (1983). Sensory masking, persistence, and enhancement in visual exploration and reading. In K. Rayner (Ed.), Eye movements in reading (pp. 41-51). New York: Academic Press.

CARPenter, P. A., \& Just, M. A. (1983). What your eyes do while your mind is reading. In $\mathrm{K}$. Rayner (Ed.), Eye movements in reading (pp. 275-307). New York: Academic Press.

CHEN, H.-C. (1982). Comprehension and memory in reading rapid, serial presentations of text. Unpublished doctoral dissertation, University of Kansas, Lawrence.

Cromer, W. (1970). The difference model: A new explanation for some reading difficulties. Journal of Educational Psychology, 61, 471-483.

Crowder, R. G. (1982). The psychology of reading: An introduction. New York: Oxford University Press.

Ehrlich, S. F., \& RAYNER, K. (1981). Contextual effects of word perception and eye movements during reading. Journal of Verbal Leaming and Verbal Behavior, 20, 641-655.
Fischler, I., \& BloOM, P. A. (1980). Rapid processing of the meaning of sentences. Memory \& Cognition, 8, 216-225.

FORSTER, K. I. (1970). Visual perception of rapidly presented word sequences of varying complexity. Perception \& Psychophysics, 8 , 215-221

GILBERT, L. C. (1959). Speed of processing visual stimuli and its relation to reading. Journal of Educational Psychology, 55, 8-14.

HochBERG, J. (1976). Toward a speech-plan eye-movement model of reading. In R. A. Monty \& J. W. Sender (Eds.), Eye movements and psychological processes (pp. 397-416). Hillsdale, NJ: Erlbaum.

Hogaboam, T. W. (1983). Reading patterns in eye movement data. In K. Rayner (Ed.), Eye movements in reading (pp. 309-332). New York: Academic Press.

Juola, J. F., Ward, N. J., \& McNamara, T. (1982). Visual search and reading of rapid, serial presentations of letter strings, words, and text. Journal of Experimental Psychology: General, 111, 208-227.

Just, M. A., \& CARPENTER, P. A. (1980). A theory of reading: From eye fixations to comprehension. Psychological Review, 87, 329-354.

KINTSCH, W., \& VAN DyJK, T. A. (1978). Toward a model of text comprehension and production. Psychological Review, 85, 363-396.

Kolers, P. A. (1983). Locations and context in eye movements. In K. Rayner (Ed.), Eye movements in reading (pp. 53-61). New York: Academic Press.

LAWRENCE, D. H. (1971). Two studies of visual search for word targets with controlled rate of presentation. Perception \& Psychophysics, 10, 85-89.

LeVy-SchoEN, A., \& O'Regan, K. (1979). The control of eye movements in reading. In P. A. Kolers, M. E. Wrolstad, \& H. Bouma (Eds.), Processing of visible language (Vol. 1, pp. 7-36). New York: Plenum Press

Martinez, P., Ghatala, E. S., \& Bell, J. A. (1980). Size of processing unit during reading and retention of prose by good and poor readers. Journal of Reading Behavior, 9, 89-95.

MAsson, M. E. J. (1983). Conceptual processing of text during skimming and rapid sequential reading. Memory \& Cognition, 11, 262-274.

MCCAlL, W. A., \& CRabss, L. M. (1951). Standard test lessons in reading. New York: Teachers College Press.

McCall, W. A., \& Crabbs, L. M. (1960). Standard test lessons in reading. New York: Teachers College Press.

MCClelland, J. L., \& O'REGAN, J. K. (1981). Expectations increase the benefit derived from parafoveal information in reading words aloud. Journal of Experimental Psychology: Human Perception and Performance, 7, 634-644.

MCConKIE, G. W. (1979). On the role and control of eye movements in reading. In P. A. Kolers, M. E. Wrolstad, \& H. Bouma (Eds.), Processing of visible language (Vol. 1, pp. 37-48). New York: Plenum Press.

MCCoNKIE, G. W. (1983). Eye movements and perception during reading. In K. Rayner (Ed.), Eye movements in reading (pp. 3-30). New York: Academic Press.

McConkIE, G. W., \& Rayner, K. (1975). The span of the effective stimulus during a fixation in reading. Perception \& Psychophysics, 17, 578-586.

McConkie, G. W., Zola, D., \& Wolverton, G. S. (1980, April). How precise is eye guidance? Paper presented at the meeting of the American Education Research Association, Boston.

Mitchell, D., \& GREeN, D. (1978). The effects of context and content on immediate processing in reading. Quarterly Journal of Experimental Psychology, 30, 609-636.

Nelson, M. J., \& DenNy, E. C. (1976). The Nelson-Denny reading test. Boston: Houghton-Mifflin.

O'REGAN, K. (1981). The "convenient viewing position" hypothesis. In D. F. Fischer, R. A. Monty, \& J. W. Senders (Eds.), Eye movements: Cognition and visual perception (pp. 289-298). Hillsdale, NJ: Erlbaum.

O'REGAN, K. J. (1983). Elementary perceptual and eye movement control processes in reading. In $\mathrm{K}$. Rayner (Ed.), Eye movements in reading (pp. 121-139). New York: Academic Press.

O'Shea, L. J., \& Sindelar, P. I. (1983). The effects of segmenting written discourse on the reading comprehension of low- and highperformance readers. Reading Research Quarterly, 18, 458-465. 
Potter, M. C. (1982, November). Very short-term memory: In one eye and out the other. Paper presented at the meeting of The Psychonomic Society, Minneapolis, MN.

PotTer, M. C. (1983). Representational buffers: The eye-mind hypothesis in picture perception, reading, and visual search. In K. Rayner (Ed.), Eye movements in reading (pp. 413-437). New York: Academic Press.

Potter, M. C., Kroll, J. F., \& Harris, C. (1980). Comprehension and memory in rapid-sequential reading. In R. Nickerson (Ed.), Attention and performance VIII (pp. 395-418). Hillsdale, NJ: Erlbaum.

RAYGOR, R. (1974). An investigation of the relationship between eye movements and comprehension in fluent reading. Unpublished doctoral dissertation, University of Minnesota, Minneapolis.

RAYNER, K. (1978). Eye movements in reading and information processing. Psychological Bulletin, 85, 618-660.

RAYNER, K. (1979). Eye movements in reading: Eye guidance and integration. In P. A. Kolers, M. E. Wrolstad, \& H. Bouma (Eds.), Processing of visible language (pp. 61-75). New York: Plenum Press.

RAYNER, K. (1983). The perceptual span and eye movement control during reading. In K. Rayner (Ed.), Eye movements in reading (pp. $97-$ 120). New York: Academic Press.

RAYNER, K., \& BERTERA, J. H. (1979). Reading without a fovea. Science, 206, 468-469.

Rayner, K., McConkie, G. W., \& Ehrlich, S. (1978). Eye movements and integrating information across fixations. Journal of $E x$ perimental Psychology: Human Perception and Performance, 4, 529-544.

RAYNer, K., MCConkIE, G. W., \& ZolA, D. (1980). Integrating in- formation across eye movements. Cognitive Psychology, 12, 202-226.

Rayner, K., Inhoff, A. W., Morrison, R. E., Slowiaczek, M. L., \& Bertera, J. H. (1981). Masking of foveal and parafoveal vision during eye fixations in reading. Journal of Experimental Psychology: Human Perception and Performance, 7, 167-179.

Rayner, K., Well, A. D., Pollatsek, A., \& Bertera, J. H. (1982). The availability of useful information to the right of fixation in reading. Perception \& Psychophysics, 31, 537-550.

RumelharT, D. E. (1977). Toward an interactive model of reading. In S. Dornic (Ed.), Attention and performance VI (pp. 573-603). Hillsdale, NJ: Erlbaum

VolkmanN, F. C. (1976). Saccadic suppression: A brief review. In R. A. Monty \& J. W. Senders (Eds.), Eye movements and psychological processes (pp. 73-83). Hillsdale, NJ: Erlbaum.

WARD, N. J. (1981). An evaluation of the effects of rapid, serial text presentations on reading comprehension. Unpublished doctoral dissertation, University of Kansas, Lawrence.

WARD, N. J., \& JUOLA, J. F. (1982). Reading with and without eye movements: A reply to Just, Carpenter, and Woolley. Journal of Experimental Psychology: General, 111, 239-241.

WOLVERTON, G. S., \& Zola, D. (1983). The temporal characteristics of visual information extraction during reading. In $\mathrm{K}$. Rayner (Ed.), Eye movements in reading (pp. 41-51). New York: Academic Press.

(Manuscript received December 30, 1983; revision accepted for publication April 13, 1984.) 